\title{
Predicting the Prognosis of Oral Tongue Carcinoma Using a Simple Quantitative Measurement Based on Preoperative MR Imaging: Tumor Thickness versus Tumor Volume
}

\author{
H. Hu, K.-L. Cheng, X.-Q. Xu, F.-Y. Wu, Y.-S. Tyan, C.-H. Tsai, and C.-Y. Shen
}

\begin{abstract}
BACKGROUND AND PURPOSE: Several studies indicated that tumor thickness or tumor volume might be helpful predictors for the prognosis of oral tongue squamous cell carcinoma. Our aim was to compare the value of tumor thickness versus tumor volume measurement based on preoperative MR imaging in predicting the prognosis of oral tongue squamous cell carcinoma, especially focusing on lymph node metastases and local recurrence.
\end{abstract}

MATERIALS AND METHODS: Clinical, pathologic, and imaging data of patients with 46 oral tongue squamous cell carcinomas were retrospectively studied. Logistic regression analysis was used to evaluate the prognostic value of tumor thickness and tumor volume based on MR imaging. Receiver operating characteristic analysis was applied for the optimal cutoff value for the identified risk variable for prognosis.

RESULTS: A higher intraclass correlation coefficient was achieved for the measurement of tumor thickness compared with tumor volume (0.990 versus 0.972$)$. Multivariate analysis showed that tumor thickness was a significant predictor of lymph node metastases $(P=.024)$, while tumor volume was not a significant predictor of either lymph node metastases or local recurrence $(P>.05)$. Receiver operating characteristic results indicated that setting a tumor thickness of $8.5 \mathrm{~mm}$ as a cutoff value could achieve the optimal diagnostic efficiency for predicting lymph node metastases (area under the curve, 0.753; sensitivity, 0.889; specificity, 0.536).

CONCLUSIONS: Tumor thickness based on preoperative MR imaging was useful in predicting the prognosis of oral tongue squamous cell carcinoma, especially lymph node metastases, in our patient population, while tumor volume was not.

ABBREVIATIONS: ROC $=$ receiver operating characteristic; $S C C=$ squamous cell carcinoma

$\mathbf{P}$

atients with oral tongue squamous cell carcinoma (SCC) are at risk of cervical lymph node metastases and local recurrence, with the risk increasing with the size and extent of tumor, defined by T stage, and influencing treatment strategy. ${ }^{1-3}$ Previous studies indicated that tumor thickness or tumor volume might also be helpful predictors for the clinical outcomes of patients with oral tongue SCC, such as cervical lymph node metastases, local recurrence, or survival rate. ${ }^{2-10}$ Which one of these 2 parameters has a better prognostic value is still unclear, however, with only a few

Received September 15, 2014; accepted after revision January 3, 2015.

From the Department of Radiology (H.H., X.-Q.X., F.-Y.W.), First Affiliated Hospital of Nanjing Medical University, Nanjing, China; Departments of Medical Imaging (K.-L.C., Y.-S.T., C.-Y.S.) and Pathology (C.-H.T.), Chung Shan Medical University Hospital, Taichung, Taiwan; School of Medical Imaging and Radiological Sciences (K.-L.C., Y.-S.T., C.-Y.S.), School of Medicine (Y.-S.T., C.-H.T., C.-Y.S.), and Institute of Medicine (C.-H.T., C.-Y.S.), Chung Shan Medical University, Taichung, Taiwan; and Department of Veterinary Medicine (K.-L.C.), National Chung Hsing University, Taichung, Taiwan.

Please address correspondence to Chao-Yu Shen, MD, Department of Medical Imaging, Chung Shan Medical University Hospital, No. 110, Sec 1, Jianguo N Rd,

Taichung, Taiwan (402); e-mail: shenchaoyu@gmail.com

http://dx.doi.org/10.3174/ajnr.A4278 studies focusing on this issue. Yuen et $\mathrm{al}^{4}$ compared the prognostic value of these 2 parameters, tumor thickness and tumor volume, together with other parameters such as tumor diameter, length, width, and area. Their measurements were based on surgical histologic specimens that were unavailable before the procedure, however, and tumor shrinkage during specimen preparation might also influence its precision in measurement.

MR imaging has been increasingly used in the preoperative evaluation of oral tongue SCCs, due to its excellent soft-tissue resolution. Preoperative MR imaging could help us to define tumor extent and volume accurately. ${ }^{11}$ Therefore, our study aimed to clarify the better predictor for the short-term prognosis of oral tongue SCCs between tumor thickness and tumor volume based on preoperative MR imaging, specifically focusing on lymph node metastases and local recurrence.

\section{MATERIALS AND METHODS Patient Population}

This study was approved by Chung Shan Medical University Hospital institutional review board. The clinical, pathologic, and preoperative MR imaging data of 223 patients with oral tongue SCC 


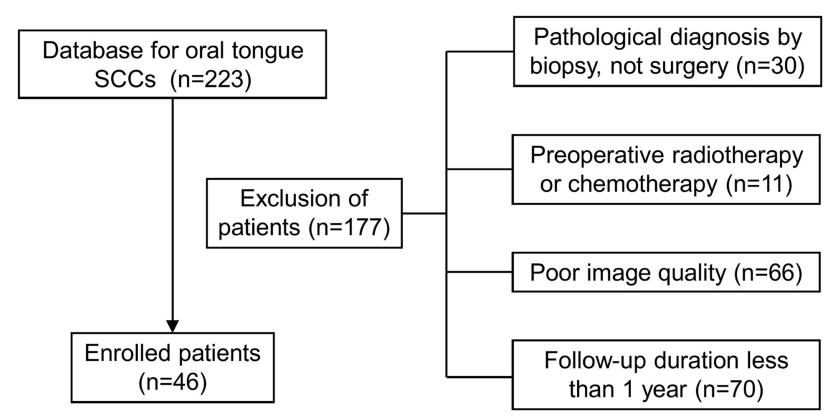

FIG 1. Study population flow chart.

during $>3$ years 6 months (February 2010 to October 2013) were retrospectively reviewed. One hundred seventy-seven patients were excluded for the following reasons: The pathologic diagnosis was established on the basis of the biopsy, not surgery $(n=30)$; radiation therapy or chemotherapy was performed before surgery $(n=11)$; poor image quality due to motion or dental artifacts $(n=66)$; and the duration of the follow-up was $<1$ year $(n=70)$. Finally, 46 patients with oral tongue SCC were enrolled in our study. The flow chart of our study population is shown in Fig 1.

The pathologic tumor stage of these 46 patients with oral tongue SCC (mean age, $51 \pm 11$ years; male/female ratio, 38:8) ranged from T1 to T4a. The mean follow-up duration was $21 \pm 10$ months. All patients underwent wide excision of the primary tumor and neck dissection. Thirty-three patients underwent supraomohyoid neck dissection, and the other 13 underwent modified radial neck dissection, with only 1 undergoing bilateral modified radial neck dissection. Seven patients received postoperative adjuvant radiation therapy, and 18 received adjuvant concurrent chemoradiotherapy. The detailed demographic features and tumor characteristics are shown in Table 1.

\section{Imaging Technique}

MR imaging was performed by using a $1.5 \mathrm{~T}$ imaging system (Signa HDxt; GE Healthcare, Milwaukee, Wisconsin) with an 8 -channel phased array neurovascular coil. Fast spin-echo sequences were used to obtain T2-weighted images (TR, 4000-7000 ms; TE, 81-90 ms; axial, coronal), T1-weighted images (TR, 450$800 \mathrm{~ms}$; TE, 13-15 ms; axial), and gadolinium contrast-enhanced T1-weighted images with fat suppression (TR, 450-800 ms; TE, 12-15 ms; axial, coronal, sagittal).

\section{Tumor Thickness and Volume Measurement}

Tumor thickness and tumor volume were measured by using axial T2WI according to methods taken from previous studies. ${ }^{3,5-7} \mathrm{Tu}-$ mor thickness was defined as the distance from the tumor surface to the deepest point of invasion. Meanwhile, after the lesion-involving area in each section was delineated by using an operatordefined ROI, the tumor volume was obtained from the sum of the areas multiplied by the section interval. The methods used for the MR imaging-based measurement of tumor thickness and tumor volume are shown in Figs 2 and 3, respectively. Imaging analysis was performed independently by 2 dedicated head and neck radiologists blinded to the pathologic results. The mean values of their measurements were calculated for further statistical analysis.

The histologic tumor thickness was also performed from the
Table 1: Patient and tumor characteristics

\begin{tabular}{|c|c|c|}
\hline Characteristics & No. of Patients & $\%$ \\
\hline Age (yr) (mean) (range) & $51(33-80)$ & \\
\hline \multicolumn{3}{|l|}{ Sex } \\
\hline Male & 38 & 83 \\
\hline Female & 8 & 17 \\
\hline \multicolumn{3}{|c|}{ Neck dissection procedure } \\
\hline SOHND & 33 & 72 \\
\hline MRND & 13 & 28 \\
\hline Bilateral MRND & 1 & 2 \\
\hline \multicolumn{3}{|l|}{ Pathologic T stage } \\
\hline $\mathrm{T} 1-2$ & 33 & 72 \\
\hline T3-4 & 13 & 28 \\
\hline \multicolumn{3}{|l|}{ Pathologic N stage } \\
\hline NO & 32 & 70 \\
\hline N1-3 & 14 & 30 \\
\hline \multicolumn{3}{|l|}{ Pathologic differentiation } \\
\hline Well & 15 & 33 \\
\hline Moderate & 27 & 59 \\
\hline Poor & 4 & 9 \\
\hline \multicolumn{3}{|l|}{ Pathologic ECS } \\
\hline Present & 10 & 22 \\
\hline Absent & 36 & 78 \\
\hline \multicolumn{3}{|l|}{ Postoperative treatment } \\
\hline RT & 7 & 15 \\
\hline CCRT & 18 & 39 \\
\hline
\end{tabular}

Note:-SOHND indicates supraomohyoid neck dissection; MRND, modified radial neck dissection; ECS, extracapsular spread; RT, radiotherapy; CCRT, concurrent chemoradiotherapy.

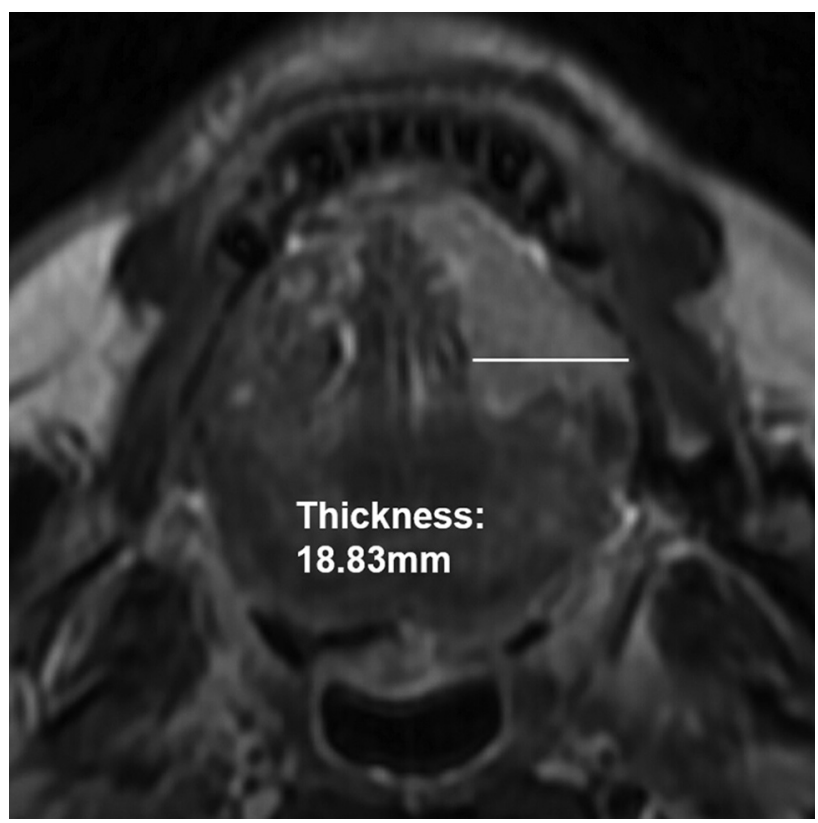

FIG 2. Tumor thickness was measured by the distance from the tumor surface to the deepest point of invasion.

tumor surface to the deepest point of invasion. Histologic tumor volume was calculated according to the previously well-documented formula ${ }^{2,12}: V=A \times B \times C / 2$. A refers to maximum tumor diameter, $B$ refers to minimum tumor diameter, and $C$ refers to the depth of tumor invasion.

\section{Prognosis}

All patients were regularly followed up by MR imaging or PET/CT examination and a clinical examination. Tongue or node biopsy 


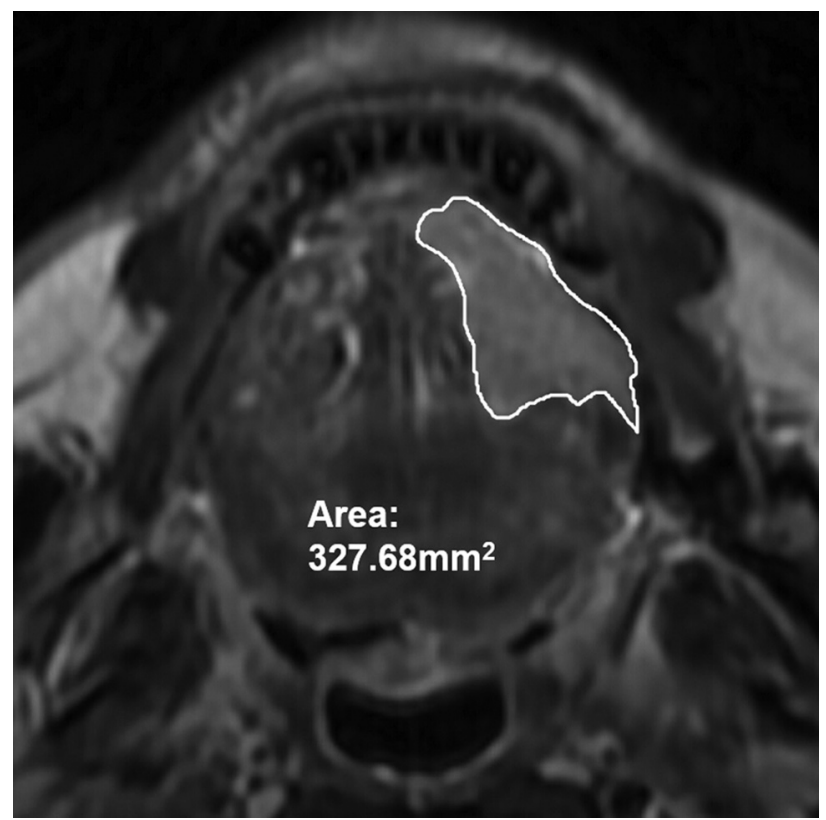

FIG 3. The lesion in each section was delineated by using an operatordefined ROI, and the volume was obtained by multiplying the entire area by the section interval.

was arranged as soon as the development of tumor local recurrence or lymph node metastasis was highly suspected after these examinations.

Lymph node metastases were defined in the following 2 situations: 1) They were confirmed pathologically from the initial surgery, and 2) no metastatic lymph node was detected during the initial surgery, and pathologically confirmed lymph node metastases occurred during the follow-up. Local recurrence was defined as a pathologically confirmed occurrence of the same malignancy at, or adjacent to, the primary tumor bed during follow-up.

\section{Statistical Analysis}

Interobserver agreement between the 2 radiologists' measurements was assessed by using the intraclass correlation coefficient. Correlations between MR imaging and histologic specimenbased measurements were assessed by using the Pearson correlation coefficient.

A univariate logistic regression analysis was initially performed to evaluate the association between each variable and lymph node metastases or local recurrence. Besides tumor thickness and tumor volume based on MR imaging, some other potential risk variables were evaluated, including sex, age, pathologic $\mathrm{T}$ stage (T1-2 or T3-4), pathologic N stage (N0 or N1-3), pathologic differentiation (well-moderate or poor), pathologic extracapsular spread (present or absent), and postoperative adjuvant modified radial neck dissection or concurrent chemoradiotherapy (yes or no). Then, all variables with a $P$ value $<.10$ in the univariate logistic regression analysis were enrolled in a multivariate logistic regression analysis model by using a forced-entry process. The odds ratios and their 95\% confidence intervals were calculated. Finally, receiver operating characteristic (ROC) analysis was applied to calculate the optimal cutoff value of the identified risk variables for prognosis. The numeric data were averaged and reported as means $\pm \mathrm{SD}$. A $P$ value $<.05$ indicated statistical significance. Statistical analysis was conducted by using SPSS 17.0 (IBM, Armonk, New York).

\section{RESULTS}

Excellent interobserver agreement was achieved for both the MR imaging-based tumor thickness and tumor volume measurement, and the tumor thickness measurement had a higher intraclass correlation coefficient value than the tumor volume measurement $(0.990$; 95\% CI, $0.965-0.997$ versus 0.972 ; $95 \% \mathrm{CI}$, 0.906-0.992). The average histologic and MR imaging tumor thicknesses were $11.7 \pm 9.7 \mathrm{~mm}$ and $13.5 \pm 9.2 \mathrm{~mm}$, respectively. The average histologic and MR imaging tumor volumes were $6.157 \pm 10.516 \mathrm{~cm}^{3}$ and $7.559 \pm 11.453 \mathrm{~cm}^{3}$, respectively. Significant correlations were found between the MR imaging-based and histologic specimen-based measurements for both tumor thickness and tumor volume $(P<.001)$.

Among the 18 patients (39\%) with lymph node metastases, pathologically positive nodes were found in 14 patients during the initial procedure, 8 of whom developed nodal metastases again during the follow-up. The other 4 patients initially had no positive lymph nodes but then developed nodal metastases during the follow-up. Concerning the univariate logistic regression analysis results, MR imaging-based tumor thickness $(P=.006)$ and pathologic extracapsular spread $(P=.003)$ had significant associations with lymph node metastases. MR imaging-based tumor volume $(P=.067)$ and pathologic T stage $(P=.057)$ showed close to significant associations, but pathologic differentiation $(P=.644)$, sex $(P=.917)$, and age $(P=.361)$ showed no statistical significance. Considering that if 2 of the independent variables were highly associated with each other, collinearity might occur, causing highly unstable estimated regression coefficients, MR imaging-based tumor thickness and volume were accepted separately into different multivariate logistic regression models, due to the strongly positive correlation between them (Pearson correlation coefficient $=0.848)$. Multivariate logistic regression analysis results showed that MR imaging-based tumor thickness and pathologic extracapsular spread were significant predictors $(P<.05)$, with MR imaging-based tumor volume and pathologic $\mathrm{T}$ stage not significant $(P>.05)$. Multivariate logistic regression analysis results for lymph node metastases are presented in Table 2.

Fourteen patients $(30 \%)$ had local recurrence during the follow-up. Four variables were found significant or close to significant in the univariate logistic regression analysis for local recurrence, including MR imaging-based tumor thickness $(P=.020)$, MR imaging-based tumor volume $(P=.066)$, pathologic $\mathrm{N}$ stage $(P=.002)$, and pathologic extracapsular spread $(P=.005)$. Pathologic T stage, pathologic differentiation, postoperative adjuvant modified radial neck dissection or concurrent chemoradiotherapy, sex, and age were not significant $(P>.1)$. Multivariate logistic regression analysis results showed that none of the variables were a significant predictor of local recurrence $(P>.05)$. Multivariate logistic regression analysis results for local recurrence are shown in Table 3.

ROC analysis indicated that the MR imaging-based tumor thickness of $8.5 \mathrm{~mm}$ might be the optimal threshold value for predicting lymph node metastases, with the area under the curve 
Table 2: Multivariate logistic regression analysis results for lymph node metastases

\begin{tabular}{lcccc}
\hline \multicolumn{1}{c}{ Variable } & $\boldsymbol{\beta}$ Coefficient & SE & Odds Ratio (95\% Cl) & $\boldsymbol{P}$ Value \\
\hline Model 1 & & & & \\
$\quad$ Pathologic ECS, present & 3.355 & 1.283 & $28.647(2.319-353.900)$ & $.009^{\mathrm{a}}$ \\
MRI tumor thickness (mm) & 0.168 & 0.074 & $1.182(1.023-1.367)$ & $.024^{\mathrm{a}}$ \\
Pathologic T stage, T3-4 & -2.118 & 1.525 & $0.120(0.006-2.391)$ & .165 \\
Model 2 & & & & \\
Pathologic ECS, present & 3.330 & 1.207 & $27.116(2.548-288.624)$ & $.006^{\mathrm{a}}$ \\
MRI tumor volume $\left(\mathrm{cm}^{3}\right)$ & 0.066 & 0.056 & $1.068(0.958-1.191)$ & .237 \\
Pathologic T stage, T3-4 & -0.679 & 1.379 & $0.507(0.034-7.562)$ & .622 \\
\hline
\end{tabular}

Note:-SE indicates standard error; ECS, extracapsular spread.

${ }^{\text {a }}$ Statistically significant

Table 3: Multivariate logistic regression analysis results for local recurrence

\begin{tabular}{lcccc}
\hline \multicolumn{1}{c}{ Variable } & $\boldsymbol{\beta}$ Coefficient & SE & Odds Ratio (95\% CI) & $\boldsymbol{P}$ Value \\
\hline Model 1 & & & & \\
$\quad$ Pathologic ECS, present & 1.110 & 1.038 & $3.035(0.397-23.211)$ & .285 \\
MRI tumor thickness (mm) & 0.060 & 0.042 & $1.061(0.978-1.152)$ & .153 \\
$\quad$ Pathologic N-stage, N1-3 & 1.273 & 0.972 & $3.571(0.531-24.019)$ & .191 \\
Model 2 & & & & \\
$\quad$ Pathologic ECS, present & 1.013 & 1.051 & $2.755(0.351-21.598)$ & .335 \\
MRI tumor volume $\left(\mathrm{cm}^{3}\right)$ & 0.045 & 0.032 & $1.046(0.983-1.112)$ & .157 \\
Pathologic N-stage, N1-3 & 1.596 & 0.952 & $4.934(0.764-31.874)$ & .094 \\
\hline
\end{tabular}

Note:-SE indicates standard error; ECS, extracapsular spread.

\section{Lymph node metastases}

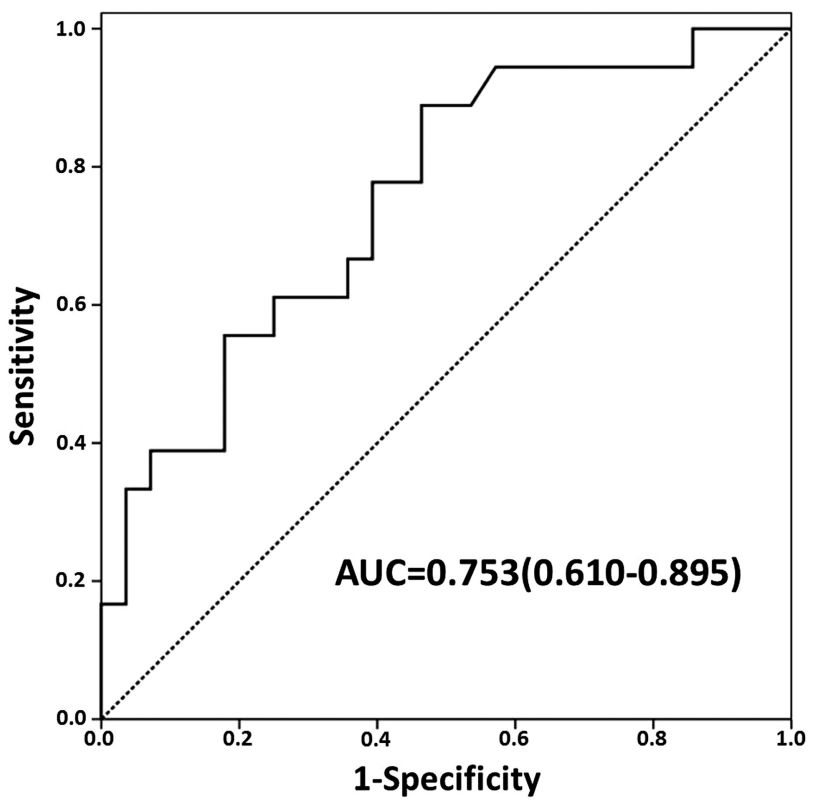

FIG 4. ROC analysis by using the measurement of tumor thickness based on MR imaging to predict lymph node metastases.

being 0.753 ; sensitivity, 0.889 ; and specificity, 0.536 . The ROC curve regarding the use of MR imaging-based tumor thickness to predict lymph node metastases is shown in Fig 4 .

\section{DISCUSSION}

The traditional prognostic model for oral tongue carcinoma was based on the anatomic details of tumor, as well as nodal and distant metastases. Using the single largest diameter as the distinction criterion for the T1 to T3 stages, however, did not always appropriately show the value of different $\mathrm{T}$ stages in relation to the oncologic outcomes. ${ }^{1,3,4}$ Additionally, the definition of the T4a stage, known as "invasion into deep (extrinsic) muscles of the tongue," seemed ambiguous. The "deep" descriptor overlooked the fact that considerable portions of extrinsic muscles lie in a very superficial plane. ${ }^{13}$ With growing evidence that tumor thickness or tumor volume might be a more accurate outcome predictor, our present study concentrated on and compared the predictive value of tumor thickness and tumor volume measurement based on preoperative MR imaging for short-term prognosis of oral tongue carcinoma, especially for lymph node metastases and local recurrence.

A higher intraclass correlation coefficient for the tumor thickness measurement based on MR imaging was noted than for tumor volume, indicating a relatively better reproducibility for tumorthickness measurement. Concerning the reasons, we suggest that the delineation of the tumor border is more complicated than the simple 2D tumor thickness measurement because of the disturbance caused by the partial volume effect, especially on the extreme tumor-imaging sections, even though the tumor was usually shown as reliably hyperintense on T2WI and not surrounded by conspicuous edema. ${ }^{5}$ This difficulty in delineation inevitably leads to greater disagreement between 2 different raters and further results in lower reproducibility.

The present study shows that the MR imaging-based tumor volume is not a significant predictor of either lymph node metastases or local recurrence, similar to the study by Yuen et al. ${ }^{4}$ They suggested that tumor thickness, not volume, is the only significant variable for the prediction of lymph node metastases, local recurrence, and survival. Nevertheless, Kuriakose et $\mathrm{al}^{2}$ suggested that tumor volume significantly correlated with lymph node metastases, after analyzing the CT images of 20 tongue cancers. Joo et $\mathrm{al}^{3}$ evaluated the MR images of 47 cases of early oral tongue cancer and also found a significant positive correlation between tumor volume and lymph node metastases. This discrepancy might be due to the different imaging modalities and the different tumor stages between these studies. Therefore, further prospective and long-term studies with the same image technique and various tumor stages are recommended to confirm the definite predictive power of tumor volume on oral tongue cancer outcomes.

Our study found that the MR imaging-based tumor thickness might be a significant predictor of lymph node metastases, which is similar to findings in previous studies. ${ }^{5-7,14}$ However, MR imaging-based tumor thickness appears unhelpful for predicting local recurrence $(P=.153)$, despite the prominently significant results from the univariate analysis $(P=.020)$. Several previous studies indicated that tumor thickness might be a potentially significant predictor of local recurrence ${ }^{4,15,16}$; however, all these previous measurements were based on histologic specimens. Measurements based on histologic specimens will inevitably be influenced by tissue shrinkage during fixation and embedding and thus should be cautiously adopted. However, no sufficiently strong evi- 
dence has been achieved by the present study, on the basis of MR imaging, to indicate the predictive significance of tumor thickness for local recurrence. We suggest that local recurrence of the primary tumor might be more influenced by additional and multiple variables. Further larger sample studies would contribute to potentially more significant results for its prediction.

Overall, MR imaging-based tumor thickness seems to be a more powerful predictor than tumor volume, especially for lymph node metastases in oral tongue carcinoma according to our study, because, as mentioned by Piazza et al, ${ }^{1}$ thicker tumor with deeper infiltration is more likely to precociously involve the lymphatic and blood vessels of tongue muscles. Subsequently, it will inevitably result in an increased risk of lymph node metastases. Moreover, tumor lesions do not always grow spherically and commonly share similar volumes but with distinct thicknesses. "Thin" tumors with larger surface diameters but smaller thickness might have a lower risk of lymph node metastases than "thick" ones having a smaller diameter but deeper infiltration. Thus, prognosis of patients might be profoundly different, even if they share the same tumor volume, with tumor thickness potentially predicting lymph node metastasis more accurately.

Using the ROC analysis, we found that an MR imaging-based tumor thickness of $8.5 \mathrm{~mm}$ might be the optimal threshold value for predicting lymph node metastases. Concerning its clinical significance, we suggest that the decision for neck dissection should be made if the tumor thickness exceeds $8.5 \mathrm{~mm}$. However, considering that the precise cutoff point varied greatly among previous studies, ${ }^{5,6,17-19}$ more accurate results should be clarified in a prospective study by using a larger sample size.

Our study has several limitations. First, only axial MR images were used to determine tumor thickness and tumor volume without applying coronal or sagittal images. However, in most previous articles, as well as in our experience, the tumor thickness and volume of most of the oral tongue carcinomas can be measured on axial images properly. Nevertheless, further studies should focus on possible differences between axial and coronal and sagittal measurements. Second, the tumor volume measurement based on a histologic specimen was not performed by using the same method as the MR imagingbased measurement, due to the constraints of our retrospective study. However, the method used to measure the histologic tumor volume has also been widely used in previous studies, ${ }^{2,12}$ with the results of our study not being intrinsically affected in our opinion. Third, we only focused on the prediction of short-term prognosis, such as lymph node metastases and local recurrence. A further study with a long-term follow-up and larger sample size would be more valuable for verifying the predictive value of identified risk factors derived from MR imaging.

\section{CONCLUSIONS}

In our study, the measurement of tumor thickness based on MR imaging was more consistent and repeatable than the measurement of tumor volume. Compared with the MR imaging-based tumor volume measurements, MR imaging-based tumor thickness demonstrated a more powerful predictive value in the prognosis of oral tongue carcinoma, mainly in predicting lymph node metastases. The ROC analysis results indicate that the optimal tumor thickness cutoff value is $8.5 \mathrm{~mm}$ for the prediction of lymph node metastases with the best sensitivity and specificity.
Our study results still need to be verified by further studies with a larger sample size and longer follow-up time.

\section{REFERENCES}

1. Piazza C, Montalto N, Paderno A, et al. Is it time to incorporate 'depth of infiltration' in the T staging of oral tongue and floor of mouth cancer? Curr Opin Otolaryngol Head Neck Surg 2014;22:81-89

2. Kuriakose MA, Loree TR, Hicks WL, et al. Tumor volume estimated by computed tomography as a predictive factor in carcinoma of the tongue. Br J Oral Maxillofac Surg 2000;38:460-65

3. Joo YH, Hwang SH, Sun DI, et al. Relationships between tumor volume and lymphatic metastasis and prognosis in early oral tongue cancer. Clin Exp Otorhinolaryngol 2013;6:243-48

4. Yuen AP, Lam KY, Wei WI, et al. A comparison of the prognostic significance of tumor diameter, length, width, thickness, area, volume, and clinicopathological features of oral tongue carcinoma. Am J Surg 2000;180:139-43

5. Preda L, Chiesa F, Calabrese L, et al. Relationship between histologic thickness of tongue carcinoma and thickness estimated from preoperative MRI. Eur Radiol 2006;16:2242-48

6. Okura M, Iida S, Aikawa T, et al. Tumor thickness and paralingual distance of coronal MR imaging predicts cervical node metastases in oral tongue carcinoma. AJNR Am J Neuroradiol 2008;29:45-50

7. Iwai H, Kyomoto R, Ha-Kawa SK, et al. Magnetic resonance determination of tumor thickness as predictive factor of cervical metastasis in oral tongue carcinoma. Laryngoscope 2002;112:457-61

8. Chen MK, Chen CM, Lee MC, et al. Primary tumor volume is an independent predictor of outcome within pT4a-staged tongue carcinoma. Ann Surg Oncol 2011;18:1447-52

9. Chew MH, Khoo JB, Chong VF, et al. Significance of tumor volume measurments in tongue cancer: a novel role in staging. ANZ J Surg 2007;77:632-37

10. Kumar T, Patel MD. Pattern of lymphatic metastasis in relation to the depth of tumor in oral tongue cancers: a clinico pathological correlation. Indian J Otolaryngol Head Neck Surg 2013;65:59-63

11. Lam P, Au-Yeung KM, Cheng PW, et al. Correlating MRI and histologic tumor thickness in the assessment of oral tongue cancer. AJR Am J Roentgenol 2004;182:803-08

12. Williams SS, Alosco TR, Mayhew E, et al. Arrest of human lung tumor xenograft growth in severe combined immunodeficient mice using doxorubicin encapsulated in sterically stabilized liposomes. Cancer Res 1993;53:3964-67

13. Boland PW, Pataridis K, Eley KA, et al. Automatic upstaging of tongue squamous cell carcinoma with lateral extrinsic muscle involvement is not justified. Int J Oral Maxillofac Surg 2013;42:1397-402

14. Bier-Laning CM, Durazo-Arvizu R, Muzaffar K, et al. Primary tumor thickness as a risk factor for contralateral cervical metastases in T1/T2 oral tongue squamous cell carcinoma. Laryngoscope 2009;119:883-88

15. Sharma $\mathrm{P}$, Shah SV, Taneja C, et al. A prospective study of prognostic factors for recurrence in early oral tongue cancer. J Clin Diaqn Res 2013;7:2559-62

16. Ganly I, Goldstein D, Carlson DL, et al. Long-term regional control and survival in patients with "low-risk," early stage oral tongue cancer managed by partial glossectomy and neck dissection without postoperative radiation: the importance of tumor thickness. Cancer 2013;119:1168-76

17. Huang TY, Hsu LP, Wen YH, et al. Predictors of locoreginal recurrence in early stage oral cavity cancer with free surgical margins. Oral Oncol 2010;46:49-55

18. Jerjes W, Upile $\mathrm{T}$, Petrie A, et al. Clinicopathological parameters, recurrence, locoregional and distant metastasis in 115 T1-T2 oral squamous cell carcinoma patients. Head Neck Oncol 2010;2:9

19. Huang SH, Hwang D, Lockwood G, et al. Predictive value of tumor thickness for cervical lymph-node involvement in squamous cell carcinoma of the oral cavity: a meta-analysis of reported studies. Cancer 2009;115:1489-97 